\title{
The Wartime Bridge Reliability Evaluation Model Based on Birth-and-Death Process
}

\author{
Duo-Dian Wang, Guo-Qing Qiu \\ Engineering Institute of Corps of Engineers, PLA University of Science \& Technology, Nanjing, China \\ E-mail: diandian2829@163.com \\ Received August 21, 2011; revised September 25, 2011; accepted October 8, 2011
}

\begin{abstract}
At first, the concept of bridge reliability is given, followed with its mathematic model. Then, based on the analysis about the mechanism of the damage and repair of bridges, and the state diversion of bridge network, the state diversion process is proved to be birth-and-death process. In the end, the state diversion balance equation of bridge network is built, and the evaluation model of wartime bridge reliability is got. The model is used in a certain example, and it is proved to be precise and credible.
\end{abstract}

Keywords: Wartime, Bridge Reliability, Evaluation, Birth-and-Death Process

\section{Introduction}

Mobile combat is the main type of the modern combat. The battlefield conditions vary from minute to minute, so maneuver rapidly or not can even decide the victory or be defeated. During the war, maneuver path is the key factor to obstruct each other's mobility. Bridges included in the maneuver paths are the main attack targets. In the paper, how to evaluate the wartime bridge reliability is researched.

The bridge reliability refers to its ability of the time in the regulated function. Under the anti-war circumstances, the bridge reliability mainly depends on its structure. On wartime, Bridge is the key factor to both parties to obstruct each other's mobility. The enemy destroys the bridge to delay mobility while we rush to repair the bridge to guarantee it.

Markov birth-and-death process method is widely apied to the research of bridge reliability. Huang Qi (see in [1]) has studied on the application of the Markov birthand-death processes in the reliability theories. Furermore, Wang Chao (see in [2]), Xia Hai-Bing (see in [3]) and Lu Ying-Zhao (see in [4]) has studied on the application of the prediction of bridge reliability. There isn't any research on the wartime bridge reliability now.

In the paper, birth-and-death process method is used to evaluate the wartime bridge reliability, combined with some related knowledge, including diagram theory, queuing theory and reliability theory. Bridge reliability is measured by the degree of reliability. Bridges are connected with each other by roads, thus, constitutes bridge network. Its scope depends on the maneuver mission. In Figure 1, bridge D-16-DM/E-17-MU is marked $b_{i}$. All the bridges of the network are involved in a bridge gather. The bridge gather is marked $B$. The reliability degree of $b_{i}$ is $r_{i}$, and the corresponding reliability degree of $B$ is $R$.

\section{The Evaluation Model of Wartime Bridge Reliability Based on the Birth-and-Death Process}

\subsection{The Mechanism of the Damage and Repair of Bridges}

Attack from enemies has regulations. Air attack is the main method. Various bridges can be sequenced according to their importance. The importance degree can be used to show how importance a bridge is, and it is marked $z_{k}$. The way to decide the importance of bridges can be found in [6,7]. The bridges can be marked $b_{k}$, and $k$ stands for its importance order. The enemy keeps stable breakage strength. Each time they attacks one target only, and just attacks the undamaged and the most important one.

Our urgent repair also has regulations. The urgent repair is carried on immediately after attack, and the allocation of soldiers commonly follows the nearby principle. If the strength of urgent repair is enough, we will rush to repair all the attacked bridges. Most of time, we have enough strength to repair the bridges after the enemies' attack. 


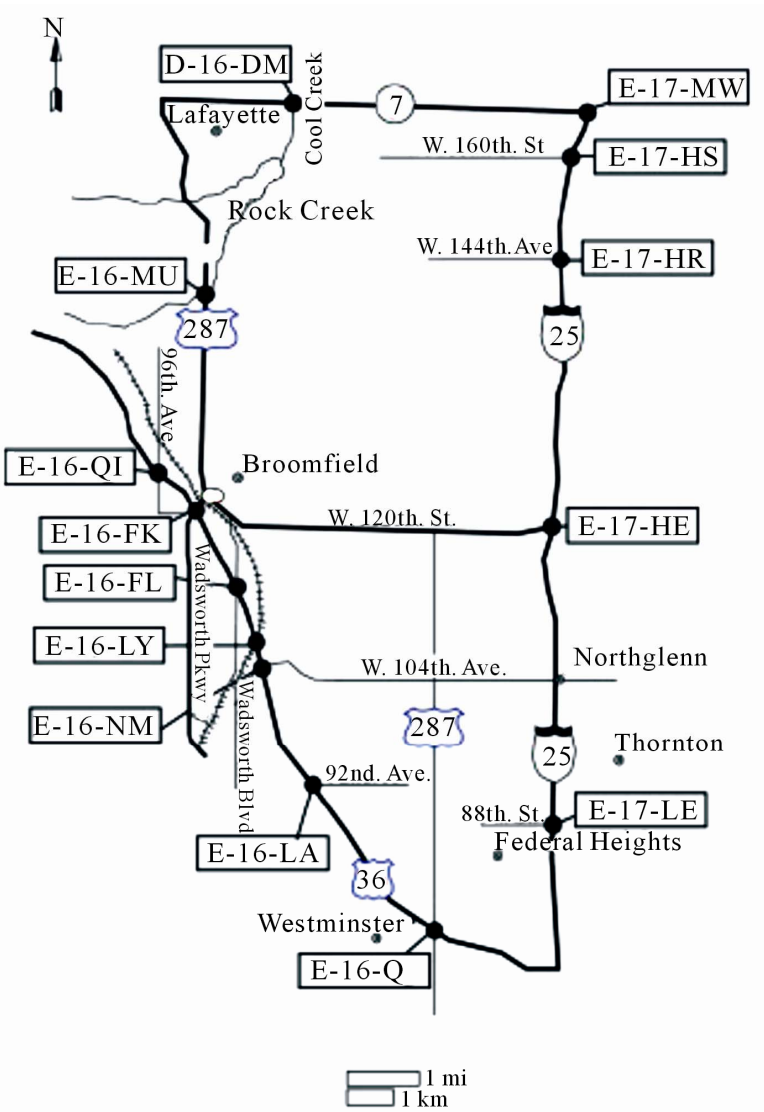

Figure 1. Bridge net of some place (see in [5]).

\subsection{The State Diversion of a Bridge}

The state of a bridge is divided into both kinds: damaged and undamaged, and is marked state 0,1 . The mechanism of state diversion of a bridge is in Figures $\mathbf{2}$ and $\mathbf{3}$.

The diversion rate from state 0 to state 1 is marked $\lambda_{k}$, and the rate from state 1 to state 0 is marked $\mu_{k} . d_{k}$ is the fought rate of a bridge , and $P_{k}$ is the broken rate ,and $x_{k}$ is the repair rate. Therefore, there is formulas as follows:

$$
\left\{\begin{array}{l}
\lambda_{k}=d_{k} \times p_{k} \\
\mu_{k}=x_{k}
\end{array}\right.
$$

The state diversion of a bridge is shown in Figure 4.

The fought rate of a bridge is decided by the power of the enemy's strike. It equals to the arrival rate of the enemy. The broken rate of a bridge refers to the rate of damage in the time of a unit (usually an hour) under average strike strength.

\subsection{The State Diversion Analysis of Bridge Network}

The process of bridge network state diversion is a typical

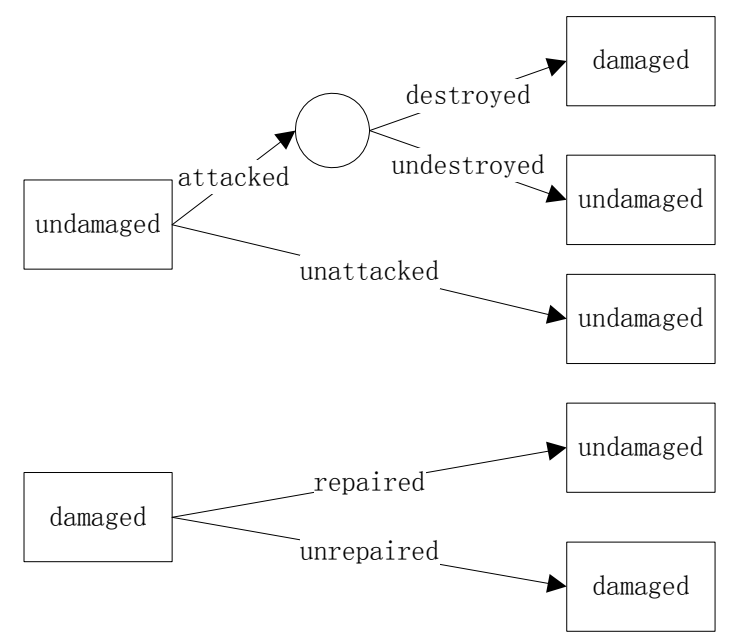

Figure 2. The mechanism of state diversion of a bridge.

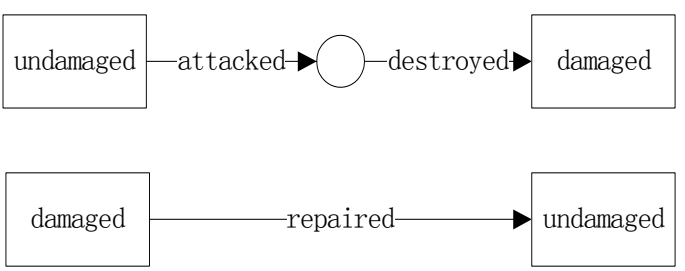

Figure 3. Simplified mechanism of state diversion of a brige.

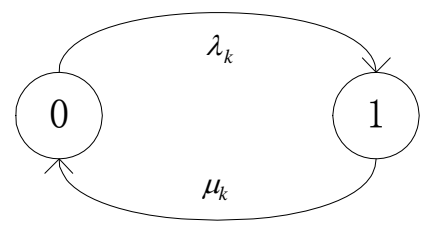

Figure 4. State diver of a bridge.

birth and death process. The information desk in the service system is the bridge network, and the customer is the enemy. The process of our urgent repair can be seen as making contributions to the customers.

The input process of birth and death process is a Poisson process; because it satisfies the following conditions (see in [8]):

1) Steadiness. In a certain time zone, there are $k$ batches of enemy called $P_{k}(t)$ to strike, which is related to the length of the time zone while it isn't any relation to the beginning of time. That is to say, the equals of $P_{k}(t)$ are the same during the time zone of $[0, t]$ or $[a, a+t]$.

2) Non after-effect. The time of the enemy coming to attack is independent during unrelated time zone. That is, in the zone $[a, a+t]$, the probability the enemy's coming isn't related to the time of that coming ahead of the time.

3) Commonness. There can be only a batch enemy in enough small time zones to arrive, and two or more batch 
to arrive in the meantime is impossible. If we use $\phi(t)$ stands for the probability of 2 or more batch to arrive between $[0, t]$, we can get $\phi(t)=o(t)(t \rightarrow 0)$.

4) Limitedness. The probability of a limited batch to arrive in arbitrarily limited time is one.

In Figure 5, state 0 refers that all the bridges are undamaged. State 1 refers that only one bridge is damaged, but others not. State k refers that k bridges are damaged, but others not. The damaged bridge in state 1 is the one with the importance $b_{1}$, the damaged bridges in state $\mathrm{k}$ are the ones with the importance of first $k$ ones, $b_{1}, b_{2}, \cdots, b_{k}$. Therefore, the probability of the state $\mathrm{k}$ is that of the bridge $b_{k}$ damaged. The reliability of bridge $b_{k}$ is that

$$
r_{k}=1-P_{k}
$$

The probability of input and output in each a state is equal, therefore, the state balance equations are as follows:

$$
\left\{\begin{array}{l}
\mu_{1} P_{1}=\lambda_{1} P_{0} \\
\lambda_{0} P_{0}+\mu_{2} P_{2}=\left(\lambda_{1}+\mu_{1}\right) P_{1} \\
\lambda_{1} P_{1}+\mu_{3} P_{3}=\left(\lambda_{2}+\mu_{2}\right) P_{2} \\
\cdots \\
\lambda_{k-2} P_{k-2}+\mu_{k} P_{k}=\left(\lambda_{k-1}+\mu_{k-1}\right) P_{k-1} \\
\lambda_{k-1} P_{k-1}+\mu_{k+1} P_{k+1}=\left(\lambda_{k}+\mu_{k}\right) P_{k}
\end{array}\right.
$$

We try to get the analytic solution of the equations, the stable state probability formulas are as follows:

$$
\left\{\begin{array}{l}
P_{k}=C_{k} P_{0} \\
C_{k}=\frac{\lambda_{k-1} \lambda_{k-2} \cdots \lambda_{0}}{\mu_{k} \mu_{k-1} \cdots \mu_{1}}
\end{array}\right.
$$

Otherwise, there is

$$
\sum_{k=0}^{K} P_{k}=\sum_{k=0}^{K} C_{k} P_{0}=1
$$

Therefore,

$$
P_{0}=\left[\sum_{k=0}^{K} C_{k}\right]^{-1}
$$

The reliability of the bridge $b_{k}$ is: $r_{k}=1-P_{k}$.

\section{Example}

The example is about the bridges in Figure $\mathbf{1}$ from reference 5 . The intention of enemy's attack is 1 time every 3 hours. The average repair time of the bridges and other parameters are in Table 1:

With the method in reference 6 , we can order the

\begin{tabular}{|c|c|c|c|c|c|c|c|c|c|c|}
\hline \multirow{2}{*}{ Number } & \multirow{2}{*}{$\begin{array}{l}\text { Bridge } \\
\text { name }\end{array}$} & \multirow{2}{*}{ Bridge type } & \multirow{2}{*}{$\begin{array}{l}\text { Number of } \\
\text { spans }\end{array}$} & \multicolumn{2}{|c|}{ Length } & \multicolumn{2}{|c|}{ Width } & \multirow{2}{*}{$\begin{array}{l}\text { Year } \\
\text { built }\end{array}$} & \multirow{2}{*}{$\begin{array}{c}\text { ADTT } \\
\text { (trucks/day) }\end{array}$} & \multirow{2}{*}{$\begin{array}{c}\text { Average repair } \\
\text { time(Hour) }\end{array}$} \\
\hline & & & & (m) & $(\mathrm{ft})$ & (m) & $(\mathrm{ft})$ & & & \\
\hline 1 & E-16-MU & Prestressed & 1 & 34.1 & 112.0 & 11.6 & 38.0 & 1994 & 810 & 1.5 \\
\hline 2 & E-16-LA & Prestressed & 2 & 77.9 & 255.5 & 39.2 & 128.5 & 1983 & 450 & 3 \\
\hline 3 & D-16-DM & Prestressed & 2 & 44.5 & 146.0 & 14.2 & 46.5 & 1990 & 390 & 1.8 \\
\hline 4 & E-16-QI & Prestressed & 2 & 74.1 & 243.2 & 30.7 & 100.7 & 1995 & 1335 & 2.8 \\
\hline 5 & E-16-LY & Prestressed & 3 & 74.3 & 243.7 & 34.1 & 112.0 & 1985 & 1610 & 2.8 \\
\hline 6 & E-16-NM & Prestressed & 2 & 64.6 & 212.0 & 28.0 & 92.0 & 1991 & 2955 & 1.5 \\
\hline 7 & E-16-MW & Prestressed & 2 & 72.7 & 238.6 & 30.5 & 100.0 & 1987 & 230 & 1.7 \\
\hline 8 & E-16-FK & Steel-beam & 4 & 69.2 & 227.0 & 10.4 & 34.0 & 1951 & 1370 & 2.5 \\
\hline 9 & E-16-FL & Steel-beam & 4 & 54.0 & 177.0 & 10.4 & 34.0 & 1951 & 765 & 2 \\
\hline 10 & E-16-Q & Steel-beam & 5 & 82.3 & 270.0 & 12.2 & 40.0 & 1953 & 890 & 3.2 \\
\hline 11 & E-17-LE & Steel plate girder & 4 & 68.6 & 225.0 & 19.7 & 64.5 & 1972 & 992 & 2.7 \\
\hline 12 & E-17-HS & Steel plate girder & 4 & 64.5 & 211.7 & 10.4 & 34.0 & 1963 & 5 & 2.5 \\
\hline 13 & E-17-HR & Steel plate girder & 4 & 64.0 & 209.8 & 10.4 & 34.0 & 1962 & 306 & 2.5 \\
\hline 14 & E-17-HE & Steel plate girder & 4 & 67.7 & 222.2 & 10.4 & 34.0 & 1962 & 1290 & 2.8 \\
\hline
\end{tabular}
bridges according to their importance.

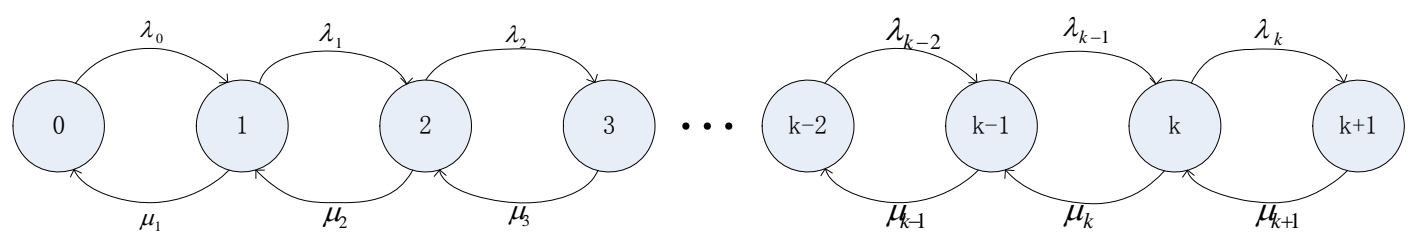

Figure 5. The state diversion of bridge network.

Table 1. Parameters of bridges in the bridge network. 
Table 2. Repair parameters of bridges in the bridge network.

\begin{tabular}{|c|c|c|c|c|c|c|c|c|c|c|c|c|c|c|}
\hline Bridge number & 1 & 2 & 3 & 4 & 5 & 6 & 7 & 8 & 9 & 10 & 11 & 12 & 13 & 14 \\
\hline Importance order $K$ & 14 & 2 & 13 & 4 & 3 & 9 & 5 & 6 & 12 & 1 & 7 & 10 & 11 & 8 \\
\hline Repair probability $\mu_{k}$ & 0.67 & 0.33 & 0.56 & 0.36 & 0.36 & 0.67 & 0.59 & 0.4 & 0.5 & 0.31 & 0.37 & 0.4 & 0.4 & 0.36 \\
\hline
\end{tabular}

Table 3. Wartime reliability of the bridges in the bridge network.

\begin{tabular}{cccccccccccccccccccccc}
\hline Order of bridge & 1 & 2 & 3 & 4 & 5 & 6 & 7 & 8 & 9 & 10 & 11 & 12 & 13 & 14 \\
\hline Importance order & 14 & 2 & 13 & 4 & 3 & 9 & 5 & 6 & 12 & 1 & 7 & 10 & 11 & 8 & \\
Reliability & $P_{k}$ & 0.9970 & 0.8516 & 0.9939 & 0.8753 & 0.8640 & 0.9768 & 0.9303 & 0.9425 & 0.9896 & 0.8516 & 0.9487 & 0.9809 & 0.9842 & 0.9530 \\
\hline
\end{tabular}

$$
\begin{aligned}
& b_{10}>b_{2}>b_{5}>b_{4}>b_{7}>b_{8}>b_{11}>b_{14}>b_{6} \\
& >b_{12}>b_{13}>b_{9}>b_{3}>b_{1}
\end{aligned}
$$

The average probability of enemy's arrival is 0.33 , the probability with that the bridges are destroyed: $\lambda_{k}=0.33$, the other parameters are in Table 2.

The state diversion balance equations are as follows:

$$
\left\{\begin{array}{l}
0.31 P_{1}=0.33 P_{0} \\
0.33 P_{0}+0.33 P_{2}=(0.33+0.31) P_{1} \\
0.33 P_{1}+0.36 P_{3}=(0.33+0.33) P_{2} \\
\cdots \\
0.33 P_{11}+0.56 P_{13}=(0.33+0.5) P_{12} \\
0.33 P_{12}+0.67 P_{14}=(0.33+0.56) P_{13}
\end{array}\right.
$$

With matlab, we solve the equations, the solutions are as follows:

$$
\begin{array}{lll}
P_{0}=0.1394 & P_{1}=0.1484 & P_{2}=0.1484 \\
P_{3}=0.1360 & P_{4}=0.1247 & P_{5}=0.0697 \\
P_{6}=0.0575 & P_{7}=0.0513 & P_{8}=0.0470 \\
P_{9}=0.0232 & P_{10}=0.0191 & P_{11}=0.0158 \\
P_{12}=0.0104 & P_{13}=0.0061 & P_{14}=0.0030
\end{array}
$$

According to the importance order, we can get the wartime reliability of the bridges in Table 3 .

Based on the result about bridge reliability in Table $\mathbf{3}$, the decide maker can get his idea about maneuver project.

\section{Conclusions}

The evaluation of wartime bridge reliability is an important content of the research about wartime mobility combat. At present, the bridge reliability research is generally limited by the peacetime appearance, while the wartime research is seldom. On the battlefield, the bridge reliability mainly depends on a series of destroy and re- pair between us and the enemy due to the abruption and quickness of the war.

In the paper, the definition of the wartime bridge reliability is given on the foundation of general bridge reliability. The state diversion of the bridge network is proved to be a birth-and-death process, and an evaluation model of the bridge reliability is got. In the future, the research will be carried on simulation experience and the improvement of the model in the text.

\section{References}

[1] Q. Huang, “Application of Markov Skeleton Processes to Reliability Theory,” Ph.D. Thesis, Middle South University, Changsha, 2004

[2] C. Wang, "Research of Markov Chain Prediction Method \& Its Application on Bridge Engineering,” Ph.D. Thesis, Beijing Jiao Tong University, Beijing, 2009

[3] H.-B. Xia, "Fuzzy reliability Forecast for Existing Bridge Based on Markov Process and Grey Theory," Modern Transportation Technology, Vol. 5, No. 2, 2008, pp. 3538

[4] Y.-Z. Lu and S.-H. He, "Prediction of Markov Fuzzy Reliability for Existing Bridge," Journal of Chang'an University: National Science Edition, Vol. 7, No. 4, 2005, pp. 39-43.

[5] F. Akgul and D. M. Frangopol, “Time-Dependent Interaction between Load Rating and Reliability of Deteriorating Bridges,” Engineering Structures, Vol. 26, No. 12, 2004, pp. 1751-1765. doi:10.1016/j.engstruct.2004.06.012

[6] W. Li, H. Jin and B. Tan, "Fuzzy Integration Judgment of Air Attack Target Selection,” Ordnance Industry Automation, Vol. 27, No. 9, 2008, pp. 25-27.

[7] W.-J. Jie and J.-L. Feng, “Air Defense Weapon Target Selection Based on Markov,” Journal of Air Force Engineering University (Natural Science Edition), Vol. 27, No. 9, 2009, pp. 37-42.

[8] J.-P. Xu, Z.-N. Hu and J. Li, “Operational Research,” Science Press, Beijing, 2008. pp. 232-235. 